\title{
Cognitive Radio MAC Protocol for WLAN
}

\section{Zhang, Qi; Fitzek, Frank H.P.; Iversen, Villy Bæk}

Published in:

19th Annual IEEE International Symposium on Personal, Indoor and Mobile Radio Communications

Link to article, DOI:

10.1109/PIMRC.2008.4699678

Publication date:

2008

Document Version

Publisher's PDF, also known as Version of record

Link back to DTU Orbit

Citation (APA):

Zhang, Q., Fitzek, F. H. P., \& Iversen, V. B. (2008). Cognitive Radio MAC Protocol for WLAN. In 19th Annual IEEE International Symposium on Personal, Indoor and Mobile Radio Communications (pp. 1-6). IEEE. https://doi.org/10.1109/PIMRC.2008.4699678

\section{General rights}

Copyright and moral rights for the publications made accessible in the public portal are retained by the authors and/or other copyright owners and it is a condition of accessing publications that users recognise and abide by the legal requirements associated with these rights.

- Users may download and print one copy of any publication from the public portal for the purpose of private study or research.

- You may not further distribute the material or use it for any profit-making activity or commercial gain

- You may freely distribute the URL identifying the publication in the public portal

If you believe that this document breaches copyright please contact us providing details, and we will remove access to the work immediately and investigate your claim. 


\section{Cognitive Radio MAC Protocol for WLAN}

\author{
Qi Zhang \\ Department of Communication, \\ Optics \& Materials \\ Technical University of Denmark \\ Lyngby, Denmark \\ Email: qz@com.dtu.dk
}

\author{
Frank H.P. Fitzek \\ Department of Communications \\ Technology \\ Aalborg University \\ Aalborg, Denmark \\ Email: ff@es.aau.dk
}

\author{
Villy B. Iversen \\ Department of Communication, \\ Optics \& Materials \\ Technical University of Denmark \\ Lyngby, Denmark \\ Email:vbi@com.dtu.dk
}

\begin{abstract}
To solve the performance degradation issue in current WLAN caused by the crowded unlicensed spectrum, we propose a cognitive radio (CR) media access protocol, C-CSMA/CA. The basic idea is that with cognitive radio techniques the WLAN devices can not only access the legacy WLAN unlicensed spectrum but opportunistically access any other under-utilized licensed spectrum without a license. The application scenario of C-CSMA/CA is infrastructure BSS (Basic Service Set) WLAN. C-CSMA/CA efficiently exploits the inherent characteristics of CSMA/CA to design distributed cooperative outband sensing to explore spectrum hole; moreover, it designs dual inband sensing scheme to detect primary user appearance. Additionally, CCSMA/CA has the advantage to effectively solve the cognitive radio self-coexistence issues in the overlapping CR BSSs scenario. It also realizes station-based dynamic resource selection and utilization. It is compatible with legacy WLAN (BSS) system. We develop and implement the simulation of C-CSMA/CA by OPNET. The simulation results show that C-CSMA/CA highly enhances throughput and reduces the queuing delay and media access delay.
\end{abstract}

\section{INTRODUCTION}

WLAN has achieved a tremendous success in recent years because of using the unlicensed spectrum. Public WLAN hotspots have been widely deployed [1]. For example, according to the reports by DataMonitor, at the end of 2003, there were approximately 31,700 such hotspots in operation globally and this number will grow at a CAGR (Compound Annual Growth Rate) of $47 \%$ to approach 146,100 by yearend 2007 [2]. However, the popularity of WLAN also gets a lot of concerns for the reason that densely spaced WLAN devices and other technology users in the unlicensed spectrum make it too crowded.

There are several research works on network congestion relief in hotspots [3], [4]. The performance of these works is limited by the available spectrum for WLAN. Therefore, to fundamentally relieve network congestion in WLAN we intend to solve spectrum scarcity issues. Cognitive radios [5][8] have emerged as a promising and key technology to solve spectrum scarcity issue for wireless applications [9]. One of the important motivation of cognitive radio is the under-utilization of licensed spectrum. That is why FCC recommended that significant spectral efficiency is expected by deploying wireless devices coexisting with the licensed users but introducing minimal interference to the licensed users [10].
Mandayam also points out one of the motivations for cognitive radio (CR) techniques is WLAN spectrum congestion and continuing density increase of wireless devices [11]. In this paper we apply CR techniques in WLAN to solve the performance degradation issue. It should be noted that the proposed scheme is different from $802.11 \mathrm{~h}$ which is single channel solution for dynamic frequency access in $5 \mathrm{GHz}$.

Knowing the aforementioned issues, this paper proposes a generic CR MAC protocol based on CSMA/CA, named as C-CSMA/CA (Cognitive radio Carrier Sensing Multiple Access / Collision Avoidance). C-CSMA/CA is designed for the infrastructure BSS (Basic Service Set), namely one AP associated with a set of WLAN stations. Infrastructure BSS is the most popular network architecture of hotspots. In CCSMA/CA, the AP can have multiple MAC_modules which can work in parallel on multiple channels. The station is assumed to work on a single channel at one time.

In cognitive radio, the user with license is defined as primary user (PU) and the user access the licensed spectrum opportunistically is defined as secondary user (SU). In this paper, PU represents any legacy user in the licensed spectrum and SU is the WLAN devices using CR techniques. From coexistence with primary user perspective C-CSMA/CA attacks two main issues: i). how to detect transmission opportunities in the unknown CR spectra; ii). how to track the usability of the known CR spectra. The first issue is solved by outband sensing. The idea of outband sensing is that the idle stations exploit the duration of the network allocation vector (NAV) to cooperatively sense an unknown potential spectrum as a way to explore a new available spectrum. For the second issue, dual inband sensing (i.e., implicit and explicit) is employed. C-CSMA/CA exploits the inherent characteristics of CSMA/CA, "listen before talk", to implicitly sense PU. Besides that, specific time $\operatorname{slot}^{1}$ is reserved for the stations to explicitly sense PU. By inband and outband sensing, the AP MAC_controller collects, manages and distributes all the unknown, unaccessible and accessible spectrum information through the AP MAC_modules. The AP MAC_controller is also responsible to allocate the accessible spectra to the AP MAC_modules. The stations update all the available spectrum information preparing for the seamless channel switch. The

\footnotetext{
${ }^{1}$ The length of the time slot depends on the PHY sensing techniques.
} 
channel switch decision is either made independently by the station (according to the station's experience or observation of the current channel status) or is triggered by channel vacation request from the AP MAC_module in case of $\mathrm{PU}$ appearance.

Besides coexistence issues with primary user, C-CSMA/CA can also deal with the issues of coexistence with the other CR users in the multiple overlapping BSSs. This is achieved by the inherent collision avoidance capability and implicit synchronization of inband sensing periods.

In brief, with cognitive radio techniques C-CSMA/CA has significant potential to improve the performance of the conventional WLAN. Furthermore, seamless primary user detection recovery, minimum interference to primary user, station-based dynamic resource selection and load balance can be realized in C-CSMA/CA.

\section{C-CSMA/CA PRotocol Description}

Generally speaking, the major difference between cognitive radio (CR) MAC and conventional MAC is that CR MAC should not only consider media share among CR users but also deal with primary user (PU) detection and protection.

The C-CSMA/CA protocol reference architecture is shown in Fig. 1. In C-CSMA/CA the media access scheme is based on the traditional CSMA/CA scheme with RTS/CTS. The key difference is that C-CSMA/CA introduces CR functions such as spectrum sensing, spectrum management and channel vacation and so on. Furthermore, with multiple MAC_modules AP can communicate with different stations on multiple channels simultaneously. In summary C-CSMA/CA inherits the distributed media access but utilizes the centralized sensing scheduling and spectrum management. In the following, the main functions in the C-CSMA/CA will be presented.

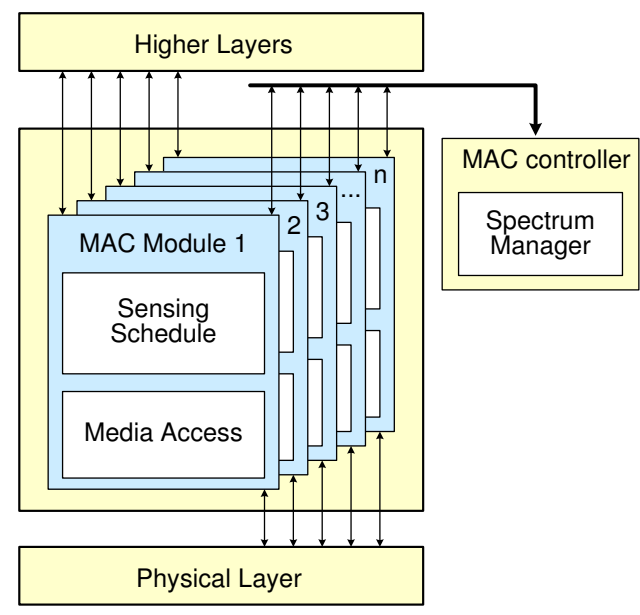

Fig. 1. Access point MAC protocol reference architecture in C-CSMA/CA.

\section{A. Primary User Detection}

Protecting primary user (PU) service not to be harmed by the interference from the secondary user is the prerequisite of cognitive radio. To fulfil with this requirement, spectrum sensing is mandatory for primary user detection purpose.

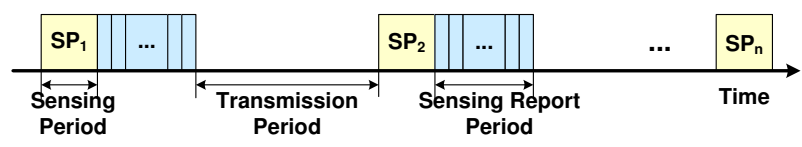

Fig. 2. Inband sensing period and transmission period

Spectrum sensing can be divided into inband sensing and outband sensing in terms of whether the sensed channel is the current channel in use.

In C-CSMA/CA the AP and its associated stations use the conventional unlicensed band to establish the network during the network initialization. Then it starts to exploit the traditional CSMA/CA characteristics to explore new usable spectrum by outband sensing. The basic idea is as following: in CSMA/CA RTS/CTS is used for media reservation. All the other devices which overhear the RTS/CTS should set the NAV timer. They will not contend the media until the NAV timer expires. In other words, the devices which do not win the media are doing nothing when waiting for the NAV timer expiration. By contrast, in C-CSMA/CA the AP MAC_module sends CTS carrying the outband sensing request to require the idle stations to perform cooperative outband sensing during the NAV duration. The outband sensing request includes the spectrum to sense and the sensing report minislots allocation. All the idle stations switch to the designated outband channel and sense the spectrum until the NAV expires. Then they switch back to the inband channel and send their sensing report beacon in report minislots based on the slotted ALOHA. By means of outband sensing, the AP obtains the channel status of an unknown channel without any additional cost in time. According to the received sensing reports, the AP will update the spectrum status which will be described in detail in Subsection II-C. Outband sensing provides the feasibility of seamless channel vacation and channel renewal.

As for the inband sensing, dual inband sensing (i.e., implicit and explicit) is used in C-CSMA/CA. It employs the inherent characteristic of the traditional CSMA/CA, "listen before talk", as implicit inband sensing. The basic idea is that if media is detected as busy, there are two possibilities: another SU activity or appearance of PU. Different from CSMA/CA, in C-CSMA/CA the stations can immediately start various PU detection process ${ }^{2}$, instead of just listening and waiting for the media becoming free. If PU is detected, SU can immediately vacate the channel. Therefore, implicit inband sensing increases the sensing frequency so that PU can be detected within the maximum interfered duration as only one packet transmission time. This is a significant advantage of C-CSMA/CA comparing with the other CR MACs such as IEEE 802.22. Besides implicit inband sensing, explicit inband sensing is also designed. The purpose of reserving specific time for periodic sensing is threefold. First, when there are only sporadic CR transmission activities, it is necessary to

\footnotetext{
${ }^{2}$ There are many primary user detection techniques such as matched filter detection, energy detection and cyclostationary feature detection, which will not be discussed in the paper.
} 
have explicit inband sensing to update the channel usability status. Secondly, the short implicit inband sensing cannot insure high precision of PU detection; furthermore, there could be interference from the SUs of another AP. Therefore, the explicit primary user detection is in need.

\section{B. Uplink and Downlink Media Access}

In conventional CSMA/CA there is no difference between uplink and downlink media access. In C-CSMA/CA the uplink media access is similar as CSMA/CA. We will not explain it in this paper. The readers who are not familiar with CSMA/CA can refer to [12]. As for the downlink, since different stations can communicate with different AP MAC_modules, the access point does not know which MAC_modules should deliver the packet to the station. How C-CSMA/CA effectively solving this issue is explained in the following.

Inspired by the operation approach in 802.11 with station sleep mode, we design the downlink media access in a similar way. The idea is that when there are data frames for the stations in the access point, the access point will broadcast a Traffic Indication MAP (TIM) message through all the active AP MAC_modules to indicate which stations have buffered traffic waiting for being picked up. The TIM is the virtual bitmap, a logical structure composed of $N$ bits. Each bit is tied to a station ID. If there is traffic buffered for that station $\mathrm{ID}$, the bit is set to 1 , otherwise, the bit is set to 0 . Once a station sees its associated bit in TIM is 1 , it will sends POLL message to retrieve its data packets from its AP MAC_module. Once the AP MAC_module receives the POLL message, it uses conventional CSMA/CA to complete the packet transmission.

\section{Spectrum Management}

The usability status of all the channels is varying over time due to PU activities. To efficiently utilize the available transmission opportunities in the CR spectrum, it is significantly important to decide when to sense which spectrum. This is realized by sensing scheduling and will be discussed in the next subsection. To design intelligent sensing scheduling, it is necessary to track the status of all the spectra. Here it is worth mentioning that although the PU activity is dynamic, we can utilize the statistical characteristics of primary user activity to reasonably estimate the mean active and idle time of primary user. The detailed study of PU traffic statistics is out of the scope of this paper. Based on this assumption the AP MAC_controller tracks spectrum status.

The AP MAC_controller regards the potential CR spectra as a channel pool and categorizes them into three classes: accessible channel (ACH), unaccessible channel (UACH), unknown channel $(\mathrm{UnCH}) . \mathrm{ACH}$ is the channel in which no $\mathrm{PU}$ is detected for the time being. UACH is the channel in which PU was detected just now. UnCH is the channel whose status is unknown to the AP or whose status in the AP has outdated. To track the channel status, each channel is associated with a timer according to its category. A TTS (Time to Sense) timer is set for periodic inband sensing for an $\mathrm{ACH}$. An UACH has a blocking timer, i.e., the AP would not ask for sensing the UACH before the blocking timer expires. For $\mathrm{UnCH}, \mathrm{AP} M A C \_c o n t r o l l e r$ has a defer_UnCH_update_timer which is designed for the distributed outband sensing and will be explained in next subsection.

Here we list the cases that the AP MAC_controller updates the channel lists:

- if primary user is detected during inband sensing, the $\mathrm{ACH}$ will be moved from the ACH list to the UACH list;

- if no primary user is detected during outband sensing, the $\mathrm{UnCH}$ will be moved the $\mathrm{UnCH}$ list to the $\mathrm{ACH}$ list, otherwise, the $\mathrm{UnCH}$ will be inserted into the UACH list;

- if an UACH blocking timer expires, the UACH will be moved from the UACH list to the UnCH list.

The spectrum management chart is shown in Fig. 3.

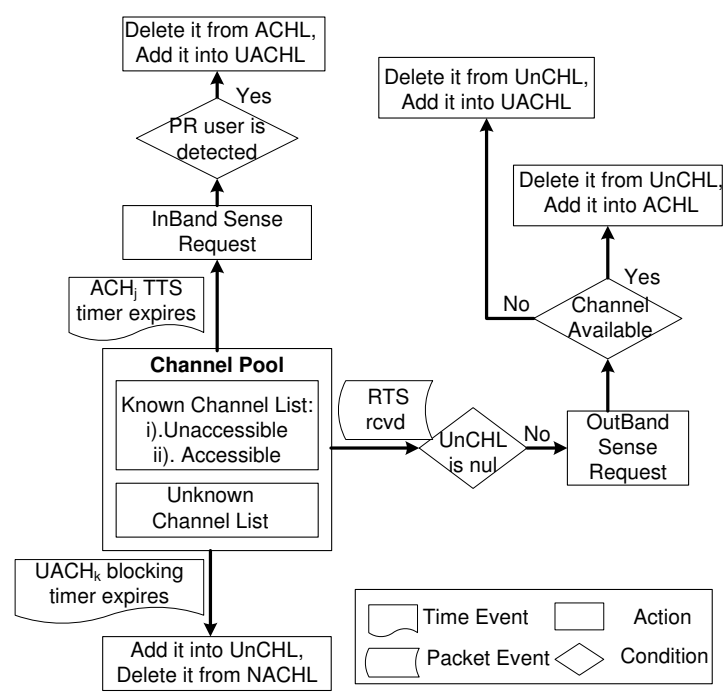

Fig. 3. Spectrum management chart

\section{Sensing Scheduling}

Sensing scheduling is one of the main additional functions in cognitive radio MAC comparing with conventional MAC. Sensing scheduling aims at answering the question of when to sense which spectrum. With the spectrum status tracking, the AP MAC_module can perform sensing scheduling.

1) Distributed Cooperative Outband Sensing: As mentioned above, C-CSMA/CA uses cooperative outband sensing to explore an unknown channel. An outband sensing request is always piggybacked in CTS from the AP MAC_module. What we would like to emphasize here is the distributed characteristics of the cooperative outband sensing. Based on the C-CSMA/CA protocol reference architecture (see Fig. 1) the AP can have parallel communications with different stations through different AP MAC_modules. Moreover, there is no synchronization among different communication pairs. It means that different AP MAC_modules receive RTSs from different stations at asynchronously. If an AP MAC_module requests sensing an $\mathrm{UnCH}$, it always takes the $\mathrm{UnCH}$ which has stayed in the $\mathrm{UnCH}$ list longest. Namely, different AP 
MAC_module select the same $\mathrm{UnCH}$ to sense unless the $\mathrm{UnCH}$ list has been updated. Thus the stations associated with different AP MAC_modules perform cooperative outband sensing and report the sensing results distributedly. Therefore, when the MAC_controller receives the first sensing result of an $\mathrm{UnCH}$ from an AP MAC_module, instead of updating the channel status immediately, it records the sensing result and starts the defer_UnCH_update_timer. The MAC_controller collects and compares the sensing results from the different AP $M A C \_m o d u l e s$ until the defer_UnCH_update_timer expires. Then the MAC_controller makes a final decision for the $\mathrm{UnCH}$ update.

An illustration of distributed cooperative outband sensing is shown in Fig. 4. Three AP MAC_modules are in active state. Each AP MAC_module associates with four stations. The AP $M A C \_$modules work on channel 1, 2 and 3, respectively. When AP $M A C \_$module 1 receives a RTS from $\mathrm{STA}_{4}^{1}$, MAC_module 1 requests the group of stations $\left(\mathrm{STA}_{i}^{1}, i=1,2,3\right)$ to cooperatively sense an unknown channel $K$. After the NAV, the stations $\left(\mathrm{STA}_{i}^{1}\right)$ send short beacons in the minislots to report the sensing results by contention ${ }^{3}$. The AP MAC_module 1 sends the collected sensing report to $M A C_{-}$controller at the end of the sensing reporting period (i.e., $t_{1}$ ). The moment $M A C_{-}$controller receives the report from AP MAC_module 1, it starts the defer_UnCH_update_timer which will expire at time $t_{u}$. AP MAC_module 2, 3 and their associated stations have the same procedure asynchronously. AP MAC_module 2, 3 send their report to MAC_controller at time $t_{2}$ and $t_{3}$, respectively. Finally the $M A C_{-}$controller makes a $\mathrm{UnCH}$ update decision at $t_{u}$ according to all the received reports.

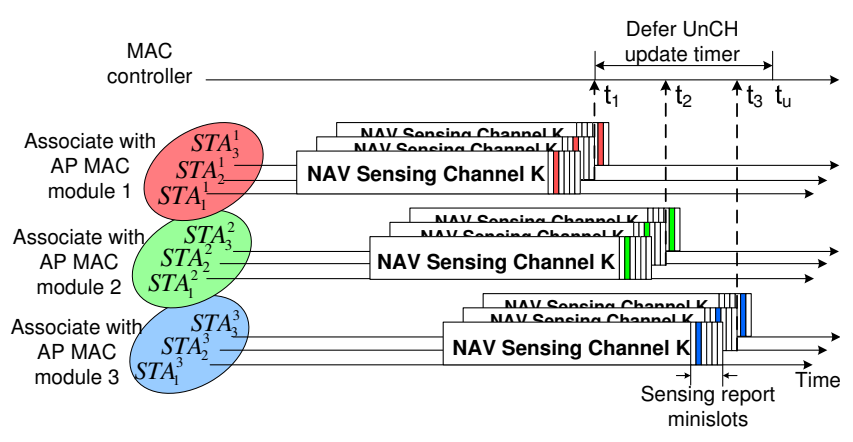

Fig. 4. Distributed outband sensing diagram

The advantage of distributed cooperative outband sensing is that it highly reduces the probability of PU miss detection. One of the reasons is that the shorter NAV duration results in the higher probability of PU miss detection, however, the distributed cooperative outband sensing virtually increases sensing time. Furthermore, more stations can join in sensing the same UnCH, which exploits spatial diversity to enhance the probability of PU detection.

\footnotetext{
${ }^{3}$ Here simple algorithm can be designed to reduce the collisions during the sensing reporting phase. For instance, the station can suppress its beacon if it hears the beacon from other station with the same sensing result.
}

2) Quasi-periodic Inband Sensing: Strict periodic inband sensing is not efficient because it often interrupts an ongoing communication. C-CSMA/CA can slightly adjust the time to perform inband sensing according to the on-going communication schedule, which is referred to as quasi-periodic inband sensing. Consequently, an inband sensing request can often be piggybacked in the traditional control packets. The advantages of quasi-periodic inband sensing has threefold: i). long time synchronization can be avoided; ii). cost for sending the inband sensing request ${ }^{4}$ is reduced; iii). it is easily to realize inband sensing synchronization among the overlapped BSSs, which is another important advantage of CCMSA/CA comparing with the other CR MACs such as IEEE 802.22 and will be addressed detailed in the next subsection.

\section{E. Self-coexistence}

Self-coexistence means coexistence of multiple overlapping cognitive radio BSSs or cells, which is another challenge of cognitive radio MAC. Multiple APs may operate with the overlapping coverage area. Furthermore, since APs may belong to different operators, explicit coordination and frequency planning cannot be assumed in cognitive radio [13].

Self-coexistence tries to solve two main issues: avoiding interference among the overlapping CR BSSs and synchronization of the overlapping inband sensing periods. In IEEE 802.22 it is quite difficult to solve these two problems. The reason lies in that all the slots usage (either transmission, reception or sensing) are allocated at the beginning of a superframe by the BS [13], however, the BS has no idea of the slots allocation of the other overlapping BSs. To attack these issues, 802.22 designs very complicated algorithms such as coexistence beacon protocol, inter-BS communication and so on which introduce big overhead and complexity (details can be referred to [13]). However C-CSMA/CA can solve these issues effectively by its inherent characteristics and the quasiperiodic inband sensing function.

C-CSMA/CA inherits the CSMA/CA characteristics that is capable to avoid interference effectively by "listen before talk", namely the devices defer transmission as long as the media is busy, no matter the media is occupied by the transmission from its own BSS or from the other BSSs. Furthermore, in some partially overlapping BSSs, parallel transmissions in different BSSs are allowed as long as there is no interference between each other.

Another important issue is the inband sensing synchronization within the overlapping BSSs, which directly affects the primary user detection. In C-CSMA/CA, once the devices within the overlapping BSSs overhear the inband sensing request from an AP MAC_module, they can immediately adjust their inband sensing schedule to synchronize with the neighbor BSS. We call this as implicit synchronization of inband sensing periods whose illustration is shown in Fig. 5. In the example, AP1 makes an inband sensing reservation (ISRv) in an CTS.

\footnotetext{
${ }^{4}$ Individual inband sensing request is sent only when there is no on-going communication and it is time to do inband sensing.
} 


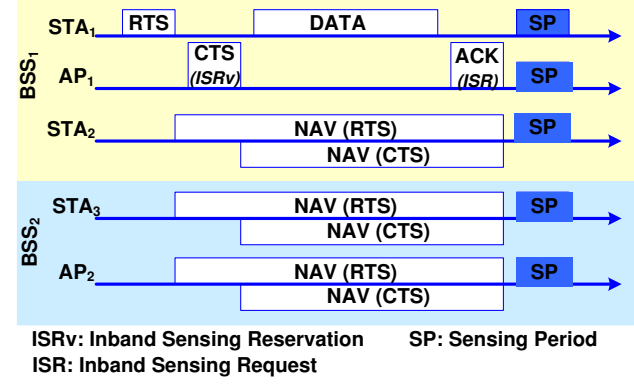

Fig. 5. Implicit synchronization of the inband sensing periods in the overlapping BSSs

Any station that receives the CTS will sense the inband channel right after the NAV timer. The inband sense request (ISR) in the ACK is for the devices who did not receive the CTS. Thus, all the devices within the overlapping area of $\mathrm{BSS}_{2}$ can synchronize their inband sensing with $\mathrm{BSS}_{1}$.

\section{F. Channel Switch and Primary User Detection Recovery}

In C-CSMA/CA, both the AP and the stations can make channel switch decision. The AP MAC_module switches channel only if PU is detected in the current channel. In this case, the AP MAC_module will send an urgent channel vacation request to its associated stations. Then AP MAC_module can switch to its backup channel or switches to the conventional WLAN spectrum or simply suspends itself. The stations have more flexible channel switch choices. They can switch to the backup channel or select any channel that another AP $M A C$ module is using. The channel switch procedure which is resulted from PU appearance is often referred to as PU detection recovery.

Besides primary user detection recovery, a station can switch to another channel if it experiences or observes a bad channel state such as low SINR (signal to interference and noise ratio), deep fading, many collisions, and so on. Such channel switch is completely spontaneous from the viewpoint of a station. By independent channel switch, it can realize station-based dynamic spectrum selection and load balance.

\section{Simulation And PerformanCE ANAlysis}

Based on the C-CSMA/CA protocol described above, we develop and implement the proposed protocol in OPNET modeler 12.0. In the simulation, the AP has four MAC_modules and one overall MAC_controller. One of the AP MAC_modules works on the conventional WLAN unlicensed spectrum. The other three AP MAC_modules can work on cognitive radio spectra as long as they detect transmission opportunities. In the simulation scenario there are 16 stations communicate with the AP. The data rate between the AP and the stations is $5 \mathrm{Mbps}$ on each channel. The explicit inband sensing is $1 \mathrm{~ms}$ and the transmission period in between is $4 \mathrm{~ms}^{5}$. Packet inter-arrival time at each station follows exponential distribution with mean

\footnotetext{
${ }^{5}$ These data is taken from IEEE 802.22. In reality, the sensing period depends on the sensing techniques.
}

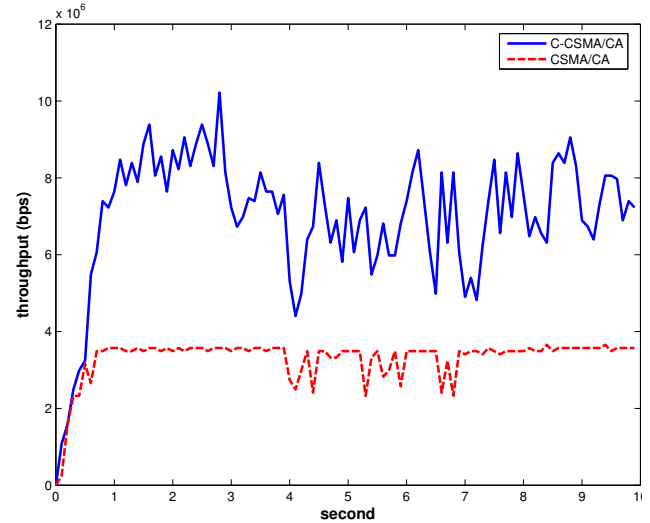

Fig. 6. Throughput comparison of C-CSMA/CA with CSMA/CA

value $10 \mathrm{~ms}$. The packet size is also exponential distribution with mean value 1024 Bytes.

In the following we compare the performance of $\mathrm{C}$ CSMA/CA with CSMA/CA in terms of throughput, queuing delay and media access delay. Fig. 6 shows the throughput comparison. It can be seen from the figure that under the assumed simulation inputs it reaches the saturation throughput using the conventional CSMA/CA. However by CR techniques C-CSMA/CA exploits the other spectrum hole to significantly enhance the throughput. With the assumption of the example, it can achieve $10 \mathrm{Mbps}$ throughput nearly as three times as that of CSMA/CA. In reality, achievable throughput depends on the number of MAC_modules and the available cognitive radio spectrum.

Fig. 7 and Fig. 8 shows the comparison of packet queuing delay and the corresponding $\mathrm{CDF}$ (cumulative distribution function). The definition of queuing delay is the time that from a packet arrival until the beginning of a transmission attempt for the packet. It is very clear that in CSMA/CA when the throughput approaches the saturation throughput, the queuing delay increases dramatically (see Fig. 7). It means that packets will be dropped if the buffer size is finite. Using C-CSMA/CA the queuing delay becomes much shorter. Fig. 8 shows that $90 \%$ packet delay is less than $0.5 \mathrm{~s}$ in CCSMA/CA, however, it reaches about $1.75 \mathrm{~s}$ in CSMA/CA. Similar comparison of media access delay is given in Fig. 9 and Fig. 10. The definition of media access delay is the time that from a transmission attempt for a packet until the packet can be successfully transmitted. The media access delay in CSMA/CA can even up to 0.16 s, however, C-CSMA/CA can reduce the media access delay to less $0.02 \mathrm{~s}$. The media access delay of $90 \%$ packets is less than $5 \mathrm{~ms}$ and $22 \mathrm{~ms}$ in C-CSMA/CA and CSMA/CA, respectively.

\section{CONClusion \& Future Work}

We propose a cognitive radio MAC protocol (C-CSMA/CA) for WLAN to solve the performance degradation issues caused by spectrum scarcity. The proposed scheme effectively exploits the inherent characteristics of the conventional CSMA/CA 


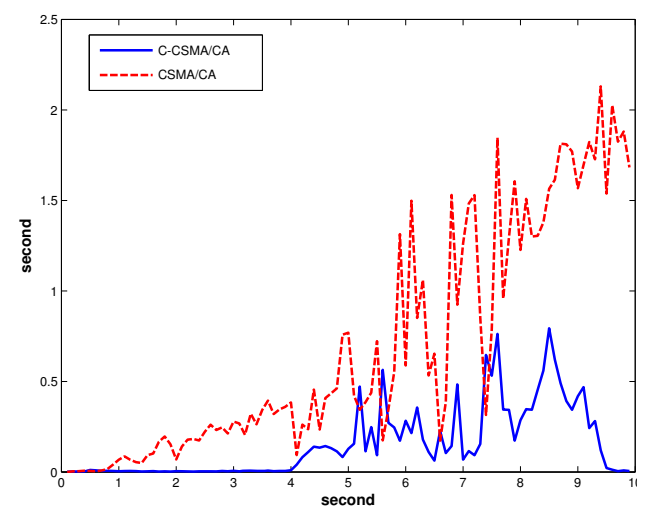

Fig. 7. Queuing delay comparison of C-CSMA/CA with CSMA/CA

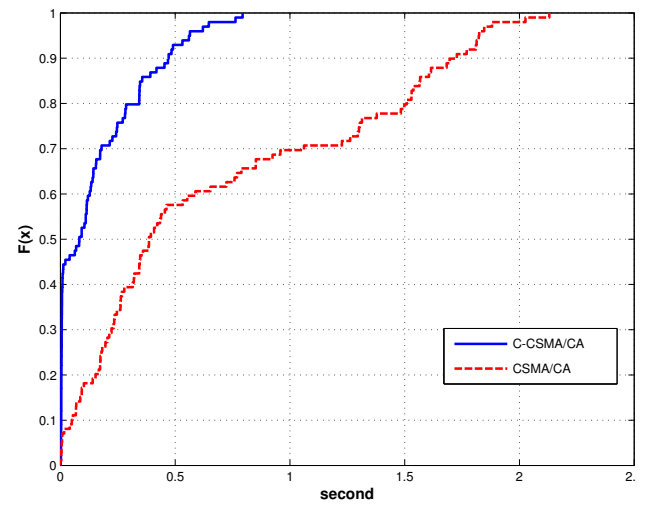

Fig. 8. Queuing delay CDF comparison of C-CSMA/CA with CSMA/CA

to facilitate its cognitive radio capability. By dual inband sensing it achieves that primary user can be detected with the interfered duration upper bound of only one packet transmission time theoretically. It can detect spectrum hole in cognitive radio spectrum by distributed cooperative outband sensing. By simulation it has shown the great advantages of C-CSMA/CA in improving the performance of WLAN. It is quite promising to implement the proposed MAC protocol with current technology.

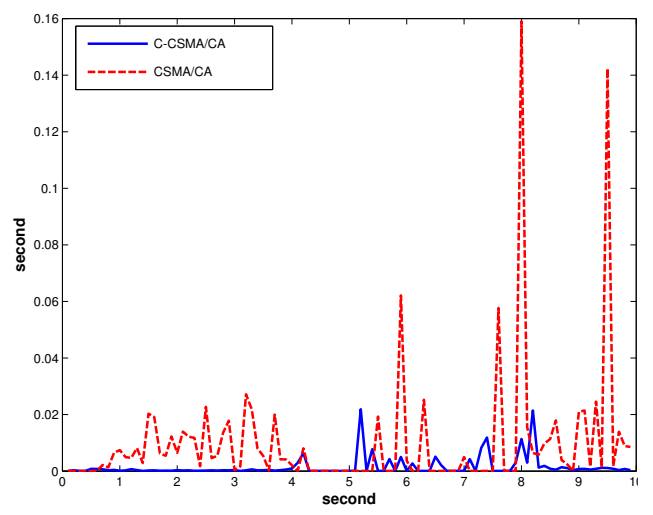

Fig. 9. Media access delay comparison of C-CSMA/CA with CSMA/CA

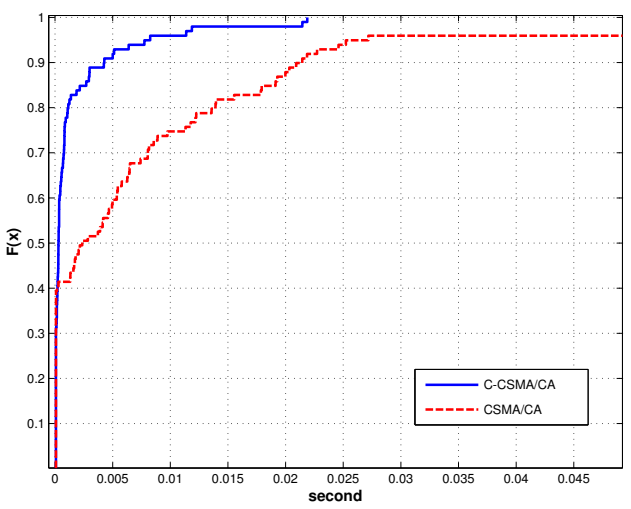

Fig. 10. Media access delay CDF comparison of C-CSMA/CA with CSMA/CA

To maximize the performance of C-CSMA/CA, it is important to find out the optimal inband sensing period and transmission period in between according to the tolerable interference of different primary users. It is also worth taking a look into the correlation of channel management timer configuration and the traffic pattern of primary users.

\section{REFERENCES}

[1] D. Qiao and S. Choi, "IEEE 802.11h: Technology and application," IT Professional, vol. 8, no. 2, pp. 43-48, March/April 2006.

[2] "Getting returns from wlans targeting niche opportunities," Datamonitor, Technical Report, Dec. 2004.

[3] O. Ekici and A. Yongacoglu, "A novel association algorithm for congestion relief in ieee 802.11 wlans," IWCMC 2006 - Proceedings of the 2006 International Wireless Communications and Mobile Computing Conference, vol. 2006, pp. 725-730, 2006.

[4] A. Balachandran, P. Bahl, and G. Voelker, "Hot-spot congestion relief in public-area wireless networks," Mobile Computing Systems and Applications, 2002. Proceedings Fourth IEEE Workshop on, pp. 70-80, 2002.

[5] J. M. et al., "Cognitive radio: making software radios more personal," IEEE Personal Communications, vol. 6, no. 4, pp. 13-18, 1999.

[6] J. Mitola, "Cognitive radio: An integrated agent architecture for software defined radio," Ph.D. dissertation, Royal Inst. Technology (KTH), Stockholm, Sweden, 2000

[7] S. Haykin, "Cognitive radio: brain-empowered wireless communications," IEEE Journal on Selected Areas in Communications, vol. 23, no. 2, pp. 201-220, 2005.

[8] I. F. Akyildiz, W.-Y. Lee, M. C. Vuran, and S. Mohanty, "Next generation/dynamic spectrum access/cognitive radio wireless networks: A survey," Computer Networks, vol. 50, no. 13, pp. 2127-2159, 2006.

[9] C. Cordeiro and K. Challapali, "C-MAC: A cognitive mac protocol for multi-channel wireless networks," New Frontiers in Dynamic Spectrum Access Networks, 2007. DySPAN 2007. 2nd IEEE International Symposium on, pp. 147-157, 2007.

[10] "Spectrum policy task force," Federal Communications Commission, Rep ET Docket no. 02-135, Nov. 2002.

[11] N. B. Mandayam, "Talk of cognitive radio networks \& the future internet," http://dimacs.rutgers.edu/Workshops/NextGenerationNetworks /slides/Mandayam.pdf, DIMACS workshop on Next Generation Networks, Rutgers University, USA., August 2007,.

[12] M. S. Gast, 802.11 Wireless Networks: The Definitive Guide, 2nd ed., ser. ISBN-10: 0596100523. O'Reilly Media,, April 25, 2005.

[13] I. . W. Group, “IEEE p802.22/d0.1 draft standard for wireless regional area networks part 22: Cognitive wireless ran medium access control (MAC) and physical layer (PHY) specifications: Policies and procedures for operation in the TV bands," Tech. Rep., May 2006 